\title{
BMJ Open Prevalence of organ failure and mortality among patients in the emergency department: a population- based cohort study
}

\author{
Peter Bank Pedersen (D , ${ }^{1}$ Daniel Pilsgaard Henriksen, ${ }^{2}$ Mikkel Brabrand, ${ }^{3}$ \\ Annmarie Touborg Lassen ${ }^{1}$
}

To cite: Pedersen PB, Henriksen DP, Brabrand M, et al. Prevalence of organ failure and mortality among patients in the emergency department: a populationbased cohort study. BMJ Open 2019;9:e032692. doi:10.1136/ bmjopen-2019-032692

\section{- Prepublication history and} additional material for this paper are available online. To view these files, please visit the journal online (http://dx.doi. org/10.1136/bmjopen-2019032692).

Received 01 July 2019 Revised 04 September 2019 Accepted 04 0ctober 2019

Check for updates

(C) Author(s) (or their employer(s)) 2019. Re-use permitted under CC BY-NC. No commercial re-use. See rights and permissions. Published by BMJ.

${ }^{1}$ Department of Emergency Medicine, Institute of Clinical Research, University of Southern Denmark, Odense University Hospital, Odense, Denmark ${ }^{2}$ Department of Clinical Biochemistry and Pharmacology, Odense University Hospital, Odense, Denmark

${ }^{3}$ Department of Emergency Medicine, Odense University Hospital \& Hospital of South West Jutland, Odense \& Esbjerg, Denmark

Correspondence to

Peter Bank Pedersen; peter.bank.pedersen@rsyd.dk

\section{ABSTRACT}

Objectives The aim was to describe population-based incidence and emergency department-based prevalence and 1-year all-cause mortality of patients with new organ failure present at arrival.

Design This was a population-based cohort study of all citizens in four municipalities (population of 230000 adults).

Setting Emergency department at Odense University Hospital, Denmark.

Participants We included all adult patients who arrived from 1 April 2012 to 31 March 2015.

Primary and secondary outcome measures Organ failure was defined as a modified Sequential Organ Failure Assessment score $\geq 2$ within six possible organ systems: cerebral, circulatory, renal, respiratory, hepatic and coagulation.

The primary outcome was prevalence of organ failure, and secondary outcomes were $0-7$ days, 8-30 days and 31-365 days all-cause mortality.

Results We identified in total 175278 contacts, of which 70399 contacts were further evaluated for organ failure. Fifty-two per cent of these were women, median age 62 (IQR 42-77) years. The incidence of new organ failure was $1342 / 100000$ person-years, corresponding to $5.2 \%$ of all emergency department contacts.

The 0-7-day, 8-30-day and 31-365-day mortality was $11.0 \%$ (95\% Cl: $10.2 \%$ to $11.8 \%$ ), $5.6 \%$ (95\% Cl: $5.1 \%$ to $6.2 \%)$ and $13.2 \%(95 \% \mathrm{Cl}: 12.3 \%$ to $14.1 \%)$, respectively, if the patient had one or more new organ failures at first contact in the observation period, compared with $1.4 \%$ (95\% Cl: $1.3 \%$ to $1.6 \%), 1.2 \%$ (95\% Cl: $1.1 \%$ to $1.3 \%$ ) and $5.2 \%$ (95\% Cl: $5.0 \%$ to $5.4 \%$ ) for patients without. Sevenday mortality ranged from hepatic failure, $6.5 \%(95 \% \mathrm{Cl}$ : $4.9 \%$ to $8.6 \%)$, to cerebral failure, $33.8 \%$ (95\% Cl: $31.0 \%$ to $36.8 \%$ ), the 8-30-day mortality ranged from cerebral failure, $3.9 \%$ (95\% Cl: $2.8 \%$ to $5.3 \%)$, to hepatic failure, $8.6 \%$ (95\% Cl: $6.6 \%$ to $10.8 \%$ ) and 31-365-day mortality ranged from cerebral failure, $9.3 \%(95 \% \mathrm{Cl}: 7.6 \%$ to $11.2 \%)$, to renal failure, $18.2 \%$ (95\% Cl: $15.5 \%$ to $21.1 \%)$. Conclusions The study revealed an incidence of new organ failure at 1342/100 000 person-years and a prevalence of $5.2 \%$ of all emergency department contacts. One-year all-cause mortality was $29.8 \%$ among organ failure patients.

\section{Strengths and limitations of this study}

- This population-based cohort study included all contacts and patients registered in the emergency department at a university teaching hospital covering a 3-year period.

- Due to the Danish comprehensive registers, we were able to present a $100 \%$ follow-up.

- The diagnosis was based on data at arrival that reflected the present clinical situation.

- It was a single-centre study, this may limit generalisability of the results.

- All minor traumas, for example, a twisted ankle, were excluded, and we had no data on organ failure among these contacts.

\section{INTRODUCTION}

\section{Background}

Patients who arrive at the doorstep of an emergency department (ED) are heterogeneous and in very different conditions where some are critically ill. Identifying these as soon as possible prehospitally as well as at arrival to the ED is crucial, as early treatment is believed to improve prognosis.

Most research regarding organ failure are on selected patient groups in the $\mathrm{ED}^{1-5}$ or in the post ED period. ${ }^{67}$ These studies find that organ failure is a frequent potential critical condition, with a high mortality.

Studies indicate a high prevalence of organ failure and in-hospital mortality. ${ }^{8-11}$ Furthermore, organ failure affects not only in-hospital prognosis but also long-term morbidity and mortality. ${ }^{12-14}$ In addition, different prevalences are described for different organ failures, and the mortality varies by organ failure and number of organ failures. ${ }^{15-17}$ These studies are less useful for the public health planning as well as upfront clinical evaluations and decisions, because patients included are selected, and might be considerable different 
from the very diverse acutely ill patients identified and treated upon arrival to the ED.

The epidemiology and characteristics for organ failure patients at population level as well as upfront at ED presentation are unknown. An efficient identification of these patients might improve quality of treatment, effective utilisation of healthcare resources and most important improve patient prognosis.

\section{Objectives}

Our primary objective was to describe the populationbased incidence and ED-based prevalence of adult patients with organ failure, and secondary end points were prevalence of specific organ failures and to examine the 7 days, $8-30$ days and $31-365$ days all-cause mortality.

\section{METHODS}

\section{Design and setting}

We performed a population-based observational follow-up study of all adult patients evaluated at the ED at Odense University Hospital, Denmark, covering a 3-year period from 1 April 2012 to 31 March 2015.

The Danish Health Care System is tax-funded and provides free of charge healthcare for the entire population. The ED at Odense University Hospital is the primary emergency entrance for a population of $\sim 230000$ covering all adult patients; except patients in chemotherapy or radiation therapy, women in active labour, patients in ongoing nephrological treatment and patients with severe cardiac disease diagnosed prehospital. The ED provides 24-hours emergency care including a level-1 trauma centre with 65000 visits per year. Odense University Hospital is a 1000-bed university teaching hospital covering all medical specialties. Primary care physicians act as gatekeepers for non-obvious acute patients and non-acute patients in the Danish Health Care System. Patients are allocated to the ED by a primary care physician or by public prehospital emergency service, ${ }^{18}$ and are evaluated by healthcare personnel, primarily four-level adaptive process triage by a specialised nurse, ${ }^{19}$ treatment or start of treatment initiated by doctors and nurses. All patients have vital values measured and laboratory tests performed based on clinical evaluation. Unaffected patients with minor trauma have none of these performed.

\section{Participants}

We retrospectively identified all acute patients $\geq 18$ years of age who arrived at the ED within the study period and evaluated patients for six possible predefined organ failures (cerebral, circulatory, renal, respiratory, hepatic or coagulation). We excluded patients, if they were living outside the hospital's primary catchment area, unidentified or registered with an invalid identification number. Follow-up was based at the Danish population-based databases ${ }^{2021}$ from initial ED contact date to emigration, death or 365 days following entry, whichever came first.

\section{Data sources}

The unique Danish personal identification number, which is assigned to all Danish citizens since 1968, and allows combination of individual patient data from different registers, was used to identify all patients. ${ }^{20}$ online Supplemental information was obtained from the Danish National Patient Registry, which contains data on all hospital admissions since 1995 in Denmark, ${ }^{21}$ and the Danish Civil Registration System. ${ }^{22}$

Vital values were extracted from electronic patient records and supplemented with data from the hospitals' laboratory. We performed a manual chart review of electronic records with missing vital values to fill in missing data.

We determined the population of adult residents in the middle study year, within the hospital catchment area, by online search at Statistics Denmark website. ${ }^{23}$

\section{Definitions}

Organ failure was defined based on Sequential Organ Failure Assessment (SOFA) Score ${ }^{24}{ }^{25}$ which is used in the intensive care unit (ICU) setting. Due to research located in an ED, we used modified definitions that were based on SOFA criteria and with input from quick-SOFA $(\mathrm{qSOFA})^{1426-30}$ :

Respiratory failure: $\mathrm{PaO}_{2}<8.4 \mathrm{kPa}$ or saturation $<91 \%$.

Coagulation failure: platelet $<100 \times 10^{9} / \mathrm{L}$ and earlier platelet count $>100 \times 10^{9} / \mathrm{L}$ or never previously registered.

Hepatic failure: bilirubin $>33 \mu \mathrm{mol} / \mathrm{L}$ and earlier bilirubin $<33 \mu \mathrm{mol} / \mathrm{L}$ or never previously registered.

Circulatory failure: systolic blood pressure $<100 \mathrm{~mm}$ $\mathrm{Hg}$.

Cerebral: Glasgow Coma Scale (GCS) Score $<13$.

Renal: creatinine $>171 \mu \mathrm{mol} / \mathrm{L}$ and earlier creatinine $<171 \mu \mathrm{mol} / \mathrm{L}$ or never previously registered.

\section{Variables}

The primary outcome was prevalence of organ failure, and secondary outcomes were 0-7 days, 8-30 days and 31-365 days all-cause mortality.

Primary variables were first recorded vital values (blood pressure, heartrate, respiratory frequency, saturation, GCS and temperature) within 6 hours after arrival to the ED. Furthermore, we included first achievable laboratory values (creatinine, $\mathrm{PaO}_{2}$, platelet, bilirubin, arterial saturation) within 24 hours of arrival. Laboratory values registered within 1 year were extracted to identify if patients had previously identified hepatic, coagulation or renal failure.

Charlson Comorbidity Index $(\mathrm{CCI})^{31}$ was calculated based on previous 10 years hospital discharge diagnoses.

\section{Statistical methods}

Descriptive data on contact and individual patient level were presented as numbers and proportions. Continuous and categorical data were presented as medians, quartiles and ranges where appropriate. Proportions were presented with $95 \%$ CI based on binomial distribution. 


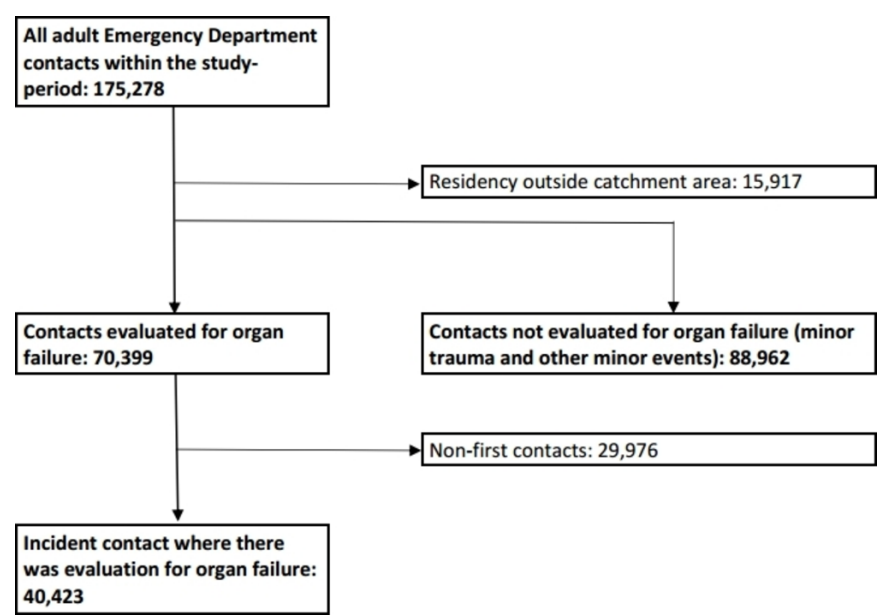

Figure 1 Flowchart of the study population, from all adult emergency department contacts to first contact patients.

Age was grouped in four: 18-44, 45-64, 65-84 and >84 years of age at arrival to the ED. CCI was calculated and presented in four groups: $0,1,2$ and $>2$.

Patients were included for post-hoc analysis the first time they presented with any new organ failure within the observation period. Incidence was determined per 100 000 person-years (py), based on the general population in middle observation year, 2013, 95\% CI for events per time unit was based at a Poisson distribution.

Data were reported as number of contacts with and without new organ failure.

Contrary to prevalence analysis that was based on all contacts evaluated for possible organ failure, mortality analysis was based at the first contact in the observation period. Mortality was presented in Kaplan-Meier plots, to illustrate any impact from numbers of organ failures, and to present any differences in mortality according to different single organ failures. The 0-7-day, 8-30day and 31-365-day mortality was presented for patients with and without new organ failure, and according to individual new organ failure and numbers of new organ failures.

To present HR with $95 \%$ CI, we performed Cox proportional hazard regression analysis, both crude analysis and adjusted for potential predefined risk factors, age, gender, comorbidity and individual organ failures.

Multivariable logistic regression adjusted for age, gender, CCI was used for risk factor evaluation of presenting with or without new organ failure.

Missing values were handled as within normal range', followed by a sensitivity analysis to evaluate this presumption, based on all-cause mortality for individual missing values.

All statistical analysis was performed using Stata V15.0.

The study was reported according to The Strengthening the Reporting of Observational Studies in Epidemiology (STROBE) statement and The STROBE explanation and elaboration. ${ }^{32} 33$

\section{Patient and public involvement}

This study was register-based without patient or public involvement in development, design or conduct of the study.

\section{RESULTS \\ Prevalence}

All ED contacts within the 3-year study period were identified, $175278,50.3 \%$ were males and the median age was 52 years (IQR 32-71) at arrival (figure 1).

We identified 9187 contacts with one or more new organ failures at arrival. This corresponds to a incidence rate of 1342/100 000 person-years (py) (95\% CI: 1066 to $1690 / 100000$ py) (supplemental 1), ${ }^{23}$ and an ED prevalence of $5.2 \%$. The most prevalent organ failure was respiratory $(n=4428,2.5 \%)$, followed by circulatory $(n=2137$, $1.2 \%)$, cerebral $(n=1593,0.9 \%)$, renal $(n=1218,0.7 \%)$, hepatic $(n=972,0.6 \%)$ and the fewest contacts presented with coagulation failure $(n=395,0.2 \%)$ (table 1$)$.

One organ failure was present in 7882 patients, two in 1092 patients and three or more in 214 patients.

Among contacts evaluated for possible new organ failure at ED arrival, $52.1 \%$ were women and median age was 62 years (IQR 42-77). Some basic characteristics are presented as online supplemental 2.

\section{Mortality}

Mortality analysis was based at the incident contact within the study period where there was evaluation for organ failure, $n=40423$, where the prevalence of new organ failure was $13.0 \%$. The most predominant risk factor for presenting with organ failure was male sex, old age and a high level of comorbidity (online supplemental $2 \& 3$ ).

The overall 7-day, 8-30-day and 31-365-day mortality was $11.0 \%$ (95\% CI: $10.2 \%$ to $11.8 \%), 5.6 \%$ (95\% CI: $5.1 \%$ to $6.2 \%)$ and $13.2 \%$ (95\% CI: $12.3 \%$ to $14.1 \%)$ if the patient had one or more new organ failures at arrival at first contact, compared with $1.4 \%$ (95\% CI: $1.3 \%$ to $1.6 \%$ ) $1.2 \%$ (95\% CI: $1.1 \%$ to $1.3 \%$ ) and $5.2 \%$ (95\% CI: $5.0 \%$ to $5.4 \%$ ) for patients without new organ failure at arrival to the ED (table 2).

The 7-day mortality was highest for patients with cerebral failure $33.8 \%$ (95\% CI: $31.0 \%$ to $36.8 \%$ ) and lowest for hepatic failure $6.5 \%$ (95\% CI: $4.9 \%$ to $8.6 \%$ ), the 8-30-day mortality was $8.6 \%$ (95\% CI: $6.6 \%$ to $10.8 \%$ ) for hepatic failure and $3.9 \%$ (95\% CI: $2.8 \%$ to $5.3 \%$ ) for cerebral failure and 31-365-day mortality was $18.2 \% \quad(95 \%$ CI: $15.5 \%$ to $21.1 \%)$ for renal failure and $9.3 \%(95 \%$ CI: $7.6 \%$ to $11.2 \%$ ) for cerebral failure. Both 7-day and 8-30-day mortality increased by number of organ failure at arrival to the ED (table 2 and figure 2).

The 0-7-day, 8-30-day and 31-365-day HR of death was 5.5 (95\% CI: 4.9 to 6.2 ), 3.2 (95\% CI: 2.7 to 3.7$)$ and 1.9 (95\% CI: 1.8 to 2.1) for patients with new organ failure at arrival compared with patients without, adjusted for gender, age and comorbidity. 
Table 1 New organ failure within study period based on all adult emergency department contacts and contacts evaluated

\section{Contacts}

In total

Age in years, median (IQR)

Gender

Female

Male

New organ failure

\begin{tabular}{|c|c|c|c|c|}
\hline Yes & 9187 & 5.2 & 9187 & 13.0 \\
\hline No & 166091 & 94.8 & 61212 & 87.0 \\
\hline \multicolumn{5}{|l|}{ Site of organ failure } \\
\hline Circulatory & 2137 & 1.2 & 2137 & 3.0 \\
\hline Coagulation & 395 & 0.2 & 395 & 0.6 \\
\hline Renal & 1218 & 0.7 & 1218 & 1.7 \\
\hline Cerebral & 1593 & 0.9 & 1593 & 2.3 \\
\hline \multicolumn{5}{|c|}{ Number of new organ failures } \\
\hline 1 & 7882 & 4.5 & 7882 & 11.2 \\
\hline 2 & 1091 & 0.6 & 1091 & 1.6 \\
\hline Infection & & & 2256 & 3.2 \\
\hline Neoplasms & & & 874 & 1.2 \\
\hline Haematologic & & & 526 & 0.8 \\
\hline Endocrine & & & 2466 & 3.5 \\
\hline Psychiatric & & & 1785 & 2.5 \\
\hline Neurologic & & & 2381 & 3.4 \\
\hline Circulatory & & & 4994 & 7.1 \\
\hline Respiratory & & & 7633 & 10.8 \\
\hline Digestive & & & 5885 & 8.4 \\
\hline
\end{tabular}

Based on patients with only one organ failure at arrival mortalities differed between the six different identified organ failures (figure 3), and the 0-7-day HR of death ranged from 2.1 (95\% CI: 1.3 to 3.3) for patients with new hepatic failure to 21.8 (95\% CI: 18.7 to 25.4 ) for patients with new cerebral failure, adjusted for gender, age and comorbidity, compared with patients without new organ failure. The adjusted 8-31-day HR ranged from coagulation 1.1 (95\% CI: 0.3 to 3.4 ) to hepatic 4.3 (95\% CI: 3.0 to
All adult ED contacts within the Contacts evaluated for possible study period organ failure

$\begin{array}{llll}\mathbf{n} & \% & \mathbf{n} & \% \\ 175278 & 100.0 & 70399 & 100.00\end{array}$

$52(32-71) \quad 62(42-77)$

$\begin{array}{llll}87160 & 49.7 & 36678 & 52.1 \\ 88118 & 50.3 & 33721 & 47.9\end{array}$

6.2), and the adjusted 31-365-day HR ranged from respiratory 1.7 (95\% CI: 1.5 to 1.9 ) to coagulation 2.4 (95\% CI: 1.6 to 3.5 ) (online Supplemental 4).

Data for patients with missing data for the individual organ failures are presented in online supplemental 5 . 
Table 2 Mortality, first contact

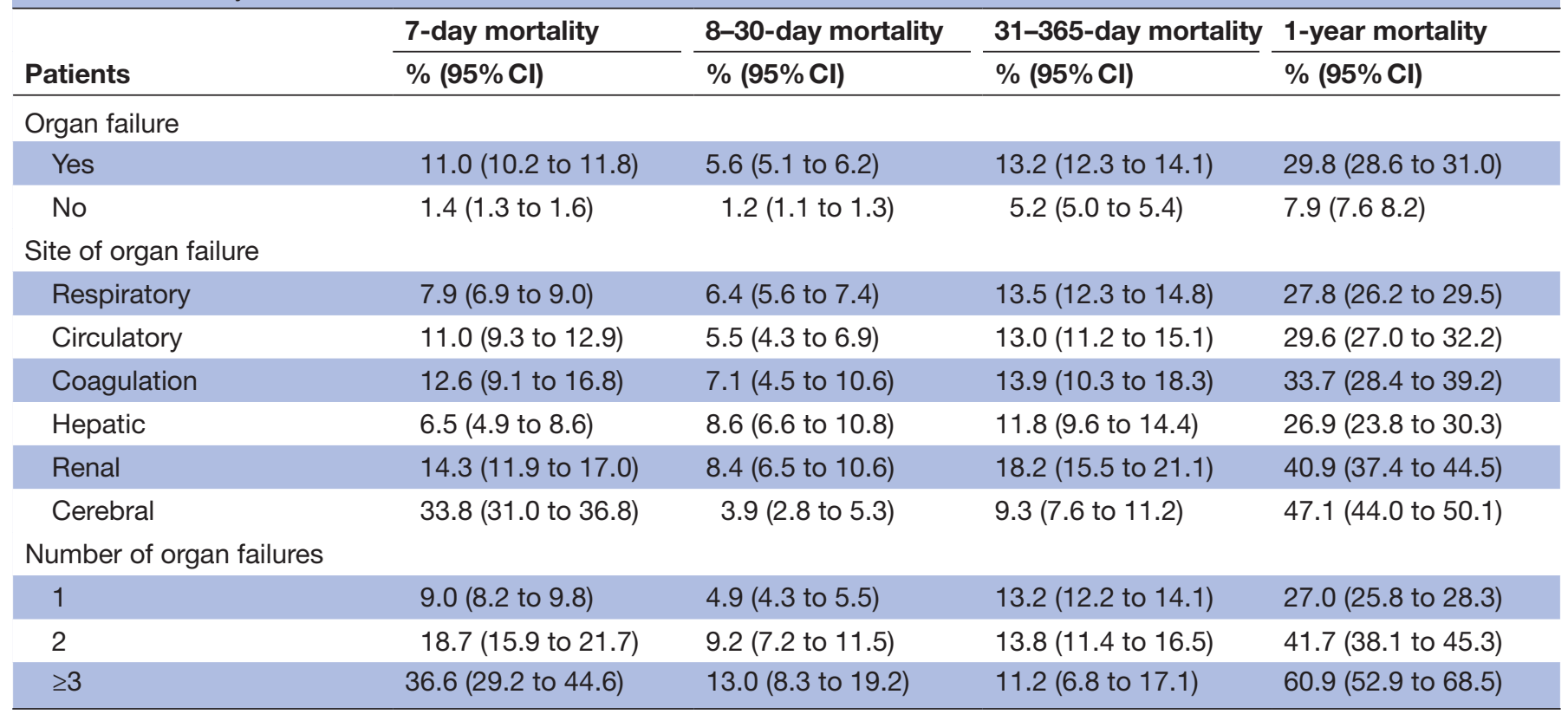

\section{DISCUSSION}

Of 175278 total contacts, we identified 9187 contacts with new organ failure at arrival to the ED at Odense University Hospital within the 3-year study period, corresponding to a prevalence of $5.2 \%$ and an incidence at population level at 1342 per 100000 person-years (py). The highest number were arriving with respiratory failure, $2.5 \%$, followed by circulatory, cerebral, renal, hepatic and coagulation.

No previous studies have presented population-based data on organ failure incidence, but studies of other time-dependent conditions report a population-based incidence of sepsis from 260-780/100 000 py, ${ }^{34-37}$ an incidence of shock at 75/100 $000 \mathrm{py},{ }^{5}$ an incidence of stroke 90-150/100 $000 \mathrm{py}^{38}{ }^{39}$ and for myocardial infarction an incidence of 210-300/100 000 py, ${ }^{41}$ all lower than the incidence of organ failure presented. This demonstrates that patients with organ failure are frequent and important as well from a public health and prehospital point of view, as well as from an ED point of view.

This study also indicates that organ failure is a serious condition with poor prognosis presenting a 0-7-day

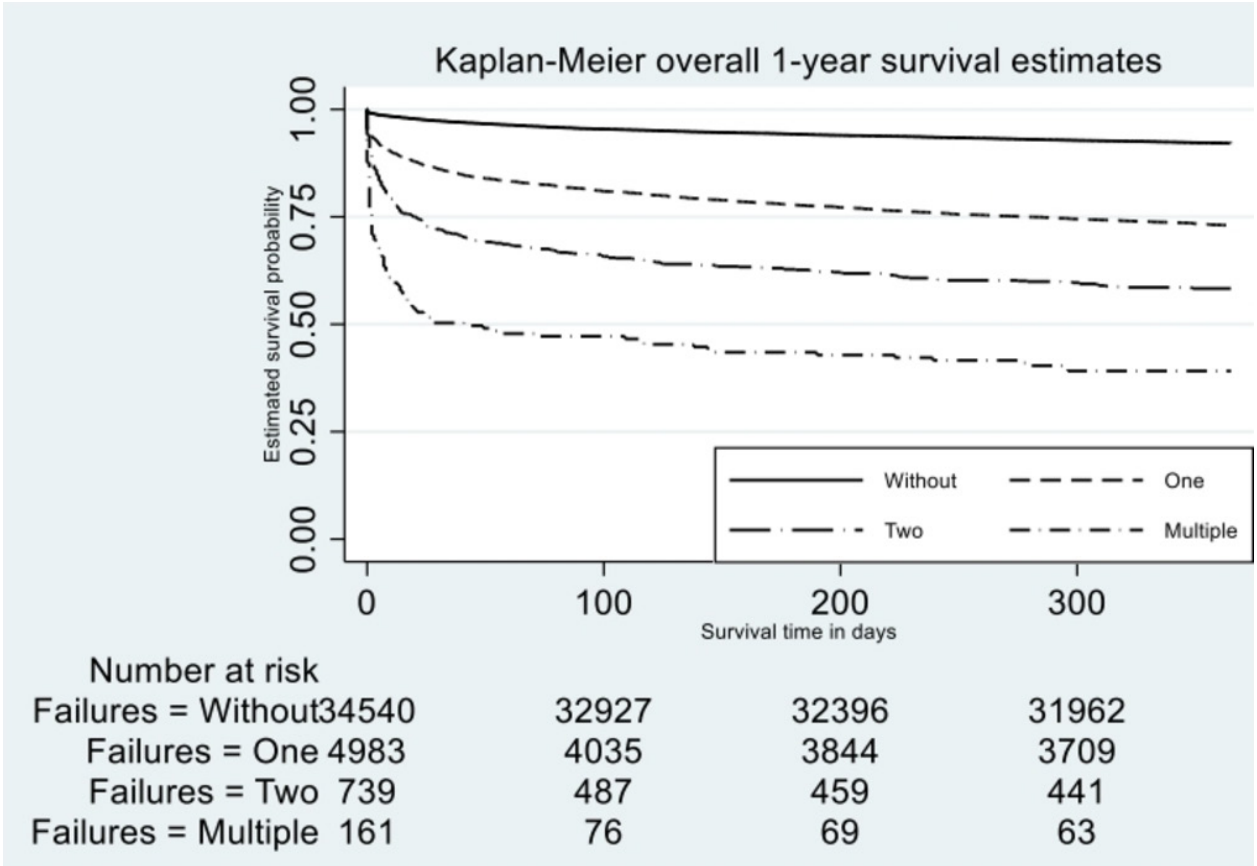

Figure 2 Kaplan-Meier survival curve illustrating overall 1-year mortality, organ failure at first contact. Without, one organ failure, two organ failures or multiple organ failures. 


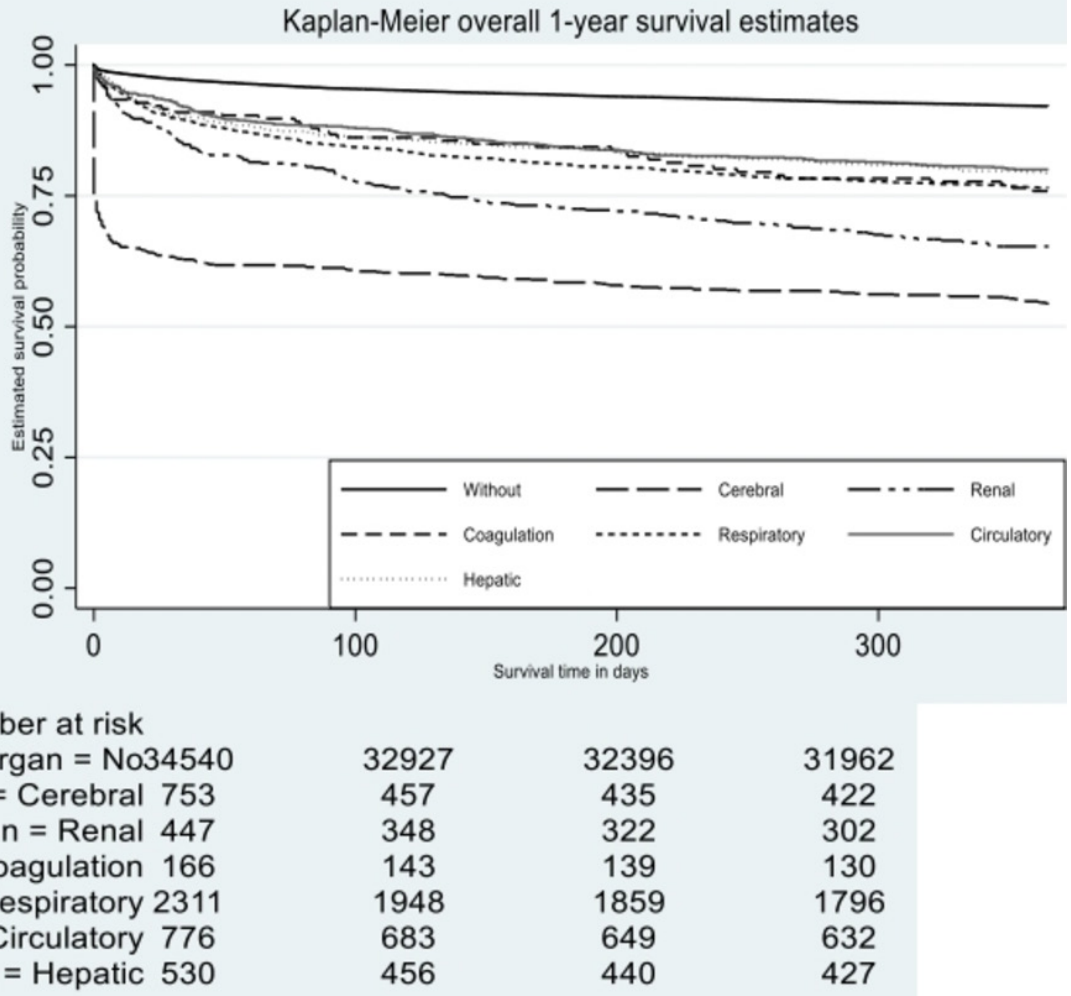

Figure 3 Kaplan-Meier survival curve illustrating overall 1-year mortality, organ failure at first contact. Without, or one single organ failure.

mortality at $11.0 \%$ for patients with organ failure at first arrival within the study period, compared with $1.4 \%$ for patients without and the numbers for 1-year all-cause mortality were $29.8 \%$ and $7.9 \%$, respectively, but the prognosis differentiates according to type of organ failure and short-time or long-time follow-up. Studies with other foci and organ failure definitions have identified in-hospital mortality at $5 \%-15 \%^{9-11}$ for patients with new organ failure, which is in accordance with our 0-7-day mortality findings. Research on more restricted patient populations in the ED, suspected infection or sepsis patients, has presented in-hospital mortality around 10\%, 30-day mortality between $10 \%$ and $25 \%$ and 1-year mortality around $30 \%$, and in parallel to the present study with mortality increasing by increasing number of organ failures. ${ }^{42}{ }^{43}$ Furthermore, cerebral failure, as in our study, had the strongest association with short-term mortality, followed by respiratory and circulatory failure, the latter in contrast to our study, and hepatic and cerebral failure were associated with long-term mortality. ${ }^{14}$ In organ failure patients with positive blood culture, the highest in-hospital mortality was observed in patients with circulatory failure followed by respiratory and cerebral. ${ }^{15}$ At the ICU, the in-hospital mortality is described around $35 \%$ and 1-year mortality around $45 \%^{713}$ which is higher than our findings, and in contrast to our numbers, hepatic failure is described with highest short-term risk of death, but in accordance with our study respiratory has the lowest short-term mortality. ${ }^{6}{ }^{13}$ Furthermore, organ failure also affects long-term prognosis, at the ICU, the 5-year mortality is described around $60 \%$ and in patients with severe organ failure around $80 \% .^{13}$ In ED patients, organ failure is described as persistent in around $30 \%$ leading to higher morbidity and healthcare burdens. ${ }^{2}$

The prevalence of organ failure identified in this study falls in between the prevalence discovered in a systematic review conducted in preparation to this study, $7 \%-23 \%$, based on very diverse non-ICU studies. ${ }^{8-11} 44$ Earlier studies indicate that the prevalence of organ failure in the ED increases by selection, where studies on patients with suspected infection present a prevalence between $20 \%$ and $28 \%,{ }^{45} 46$ and the prevalence is reported at almost $60 \%$ among patients with verified infection. ${ }^{47}$ It seems more unclear when looking at studies focusing at sepsis, before the third definition based on organ failure, where the prevalence of organ failure in the ED is described between $50 \%$ and $80 \%{ }^{48} 49$

Research on patients at the ICU has revealed a prevalence of organ failure between $35 \%$ and $60 \%,{ }^{6712}$ that is between three and five times the prevalence explored by our study, reflecting that patients at the ICU are highly selected and in worse condition, according to organ failure, than patients at arrival at the ED.

Studies describe respiratory and circulatory failure as most prevalent organ failures at the ICU, ${ }^{67}$ and this is in accordance with our findings at arrival to an ED.

Research on organ failure in non-ICU settings on infected, and shock patients, reveals the highest numbers of organ failure to be cardiovascular ${ }^{5}$ followed by either respiratory or renal, ${ }^{17} 46$ and the fewest patients with 
organ failure presented with hepatic failure, ${ }^{250}$ almost in accordance with our findings.

Definitions of organ failure has major impact on the results presented, and one needs to consider this fact, when comparing data from organ failure studies using different definitions. Earlier studies have used, for example, discharge diagnoses, single organ failure scoring system or modified 2001 sepsis consensus organ failure definitions. ${ }^{8-11}{ }^{51}$ Recently, most studies present organ failure data based on the SOFA Score,${ }^{2425}$ but some of the values are not clinical applicable to the $\mathrm{ED}$, and this have led to almost equal attempts to modify the SOFA Score to fit the different ED settings in studies conducted. ${ }^{42} 5253$ These approaches are almost identical to our modified organ failure definitions, but the circulatory and respiratory differs slightly from study to study due to differences in accessible information in different ED settings. In our case, we were not able to extract information on oxygen supplementation or intravenous fluid consumption. Organ failure is defined as a SOFA Score $\geq 2^{2425}$ but we wanted to present data on single organ failure and not on combined scores from different organs, and this and abovementioned led to the modified organ failure definitions.

\section{Strengths}

Our study has several strengths. The cohort includes all contacts and patients registered in the ED at a university teaching hospital over a 3-year period. This provides a very broad and diverse group of acutely ill contacts and patients.

To fill in missing data, we performed a manual review of the patient's electronic records, to minimise risk of bias before calculating the final estimations of organ failure prevalence and prognosis.

The diagnosis is based on data at arrival that reflects the present clinical situation.

Due to clearly defined catchment area, and one hospital serving this area, we were able to estimate populationbased incidence.

Due to the Danish comprehensive registers, we were able to present a $100 \%$ follow-up.

\section{Limitations}

The study has some limitations especially regarding generalisability. It is a single-centre study. The results are probably generalisable to other populations and ED settings that treat acutely ill adult patients at arrival, and where the organisations resemble the description outlined in the Methods section.

Furthermore, missing data are present, although we did an extensive job to minimise the risk of bias attributed to missing data, we still present between $10 \%$ and $30 \%$ missing data on individual parameters, and the mortality data indicated that missing data was not equal to within normal range', this might result in an underestimation of prevalence of new onset organ failure and the consequent mortality.
Finally, some patients might have had an organ failure diagnosed by their general practitioner which the study did not account for.

We decided to exclude all minor trauma for example, a twisted ankle, but we have no data on organ failure among these contacts. We do believe that these contacts were without new organ failure, but we cannot be $100 \%$ certain, and if some of these contacts arrived with a new organ failure, our prevalences are underestimated, we are presenting a minimum of organ failure prevalence.

\section{Perspectives}

This study describes organ failure as both frequent and critical when present at arrival to an ED and indicates that we need to stay focused at acutely ill adult patients in these states of disorders. This highlights the need for awareness regarding organ failure as soon as possible after arrival, or even earlier, in the prehospital setting. Healthcare personnel need to recognise the signs of organ failure, even before they become obvious, because presence of clinically recognisable signs of organ failure results in better treatment and compliance to guidelines. ${ }^{54}$ More research on aetiologies, signs of organ failure, and where and when to start treatment is needed, to hopefully better the poor prognosis for organ failure patients. Furthermore, established organ failure definitions applicable to different emergency settings are needed to improve quality of organ failure research in the emergency settings in the future.

\section{CONCLUSION}

Organ failure was both frequent and a serious condition when present in adult acutely ill patients at arrival to an ED. We present an incidence of 1342/100 000 personyears and a 1-year all-cause mortality of $29.8 \%$ with wide variation according to type of organ failure.

\section{Twitter Daniel Pilsgaard Henriksen @dphdk}

Acknowledgements Data from this study were used in a Poster Presentation at the 39th International Symposium on Intensive Care and Emergency Medicine in Brussels, Belgium, 19-22 March 2019.

Contributors PBP, ATL, MB and DPH contributed to the development of this study and wrote the paper. PBP analysed the data with input from DPH. All authors have read and approved the final manuscript.

Funding This research received no specific grant from any funding agency in the public, commercial or not-for-profit sectors. PBP was supported by the University of Southern Denmark, the Region of Southern Denmark, King Christian X Foundation and Torben \& Alice Frimodts Foundation, but they were not involved in any aspect of this study and had no influence on the study results or publication, and no role in study design, data collection or analysis. ATL received an unrestricted grant from the private philanthropic fund TrygFonden given to the University of Southern Denmark.

Competing interests None declared.

Patient consent for publication Not required.

Ethics approval The study was approved by the Danish Health and Medicines Authority (J No 3-3013-1070/1) and the Danish Data Protection Agency (J No 2008-58-0035) in compliance with Danish law. No further approval is required for register-based studies in Denmark.

Provenance and peer review Not commissioned; externally peer reviewed. 
Data availability statement Data are available upon reasonable request.

Open access This is an open access article distributed in accordance with the Creative Commons Attribution Non Commercial (CC BY-NC 4.0) license, which permits others to distribute, remix, adapt, build upon this work non-commercially, and license their derivative works on different terms, provided the original work is properly cited, appropriate credit is given, any changes made indicated, and the use is non-commercial. See: http://creativecommons.org/licenses/by-nc/4.0/.

ORCID iD

Peter Bank Pedersen http://orcid.org/0000-0002-2831-674X

\section{REFERENCES}

1 Chen Y-X, Li C-S. Risk stratification and prognostic performance of the predisposition, infection, response, and organ dysfunction (PIRO) scoring system in septic patients in the emergency department: a cohort study. Crit Care 2014;18.

2 Guirgis FW, Brakenridge S, Sutchu S, et al. The long-term burden of severe sepsis and septic shock: sepsis recidivism and organ dysfunction. J Trauma Acute Care Surg 2016;81:525-32.

3 Jessen MK, Skibsted S, Shapiro NI. Number of organ dysfunctions predicts mortality in emergency department patients with suspected infection: a multicenter validation study. Eur J Emerg Med 2017;24:176-82.

4 Williams JM, Greenslade JH, McKenzie JV, et al. Systemic inflammatory response syndrome, quick sequential organ function assessment, and organ dysfunction: insights from a prospective database of ED patients with infection. Chest 2017;151:586-96.

5 Holler JG, Henriksen DP, Mikkelsen S, et al. Erratum to: Shock in the emergency department; a 12 year population based cohort study. Scand J Trauma Resusc Emerg Med 2017;25:103.

6 Bingold TM, Lefering R, Zacharowski K, et al. Individual Organ Failure and Concomitant Risk of Mortality Differs According to the Type of Admission to ICU - A Retrospective Study of SOFA Score of 23,795 Patients. PLoS One 2015;10:e0134329.

7 Sakr Y, Lobo SM, Moreno RP, et al. Patterns and early evolution of organ failure in the intensive care unit and their relation to outcome. Crit Care 2012;16.

8 Lindvig KP, Henriksen DP, Nielsen SL, et al. How do bacteraemic patients present to the emergency department and what is the diagnostic validity of the clinical parameters; temperature, C-reactive protein and systemic inflammatory response syndrome? Scand $J$ Trauma Resusc Emerg Med 2014;22.

9 Benns M, Carr B, Kallan MJ, et al. Benchmarking the incidence of organ failure after injury at trauma centers and nontrauma centers in the United States. J Trauma Acute Care Surg 2013;75:426-31.

10 Challiner R, Ritchie JP, Fullwood C, et al. Incidence and consequence of acute kidney injury in unselected emergency admissions to a large acute UK Hospital trust. BMC Nephrol 2014;15:84.

11 Churpek MM, Zadravecz FJ, Winslow C, et al. Incidence and prognostic value of the systemic inflammatory response syndrome and organ dysfunctions in ward patients. Am J Respir Crit Care Med 2015;192:958-64.

12 Ranzani OT, Zampieri FG, Besen BAMP, et al. One-Year survival and resource use after critical illness: impact of organ failure and residua organ dysfunction in a cohort study in Brazil. Critical Care 2015;19.

13 Lone NI, Walsh TS. Impact of intensive care unit organ failures on mortality during the five years after a critical illness. Am J Respir Crit Care Med 2012;186:640-7.

14 Schuler A, Wulf DA, Lu Y, et al. The impact of acute organ dysfunction on long-term survival in sepsis, 1530.

15 Dat VQ, Long NT, Hieu VN, et al. Clinical characteristics, organ failure, inflammatory markers and prediction of mortality in patients with community acquired bloodstream infection. BMC Infect Dis 2018;18:535.

16 Ranzani OT, Shankar-Hari M, Harrison DA, et al. A comparison of mortality from sepsis in Brazil and England: the impact of heterogeneity in general and Sepsis-Specific patient characteristics. Critical care medicine 2018.

17 Rohde JM, Odden AJ, Bonham C, et al. The epidemiology of acute organ system dysfunction from severe sepsis outside of the intensive care unit. J Hosp Med 2013;8:243-7.

18 Mikkelsen S, Krüger AJ, Zwisler ST, et al. Outcome following physician supervised prehospital resuscitation: a retrospective study. BMJ Open 2015;5:e006167.

19 Lindberg Søren Østergaard, Lerche la Cour J, Folkestad L, et al. The use of triage in Danish emergency departments. Dan Med Bull 2011;58:A4301.
20 Frank L, Epidemiology FL. Epidemiology. when an entire country is a cohort. Science 2000;287:2398-9.

21 Schmidt M, Schmidt SAJ, Sandegaard JL, et al. The Danish national patient registry: a review of content, data quality, and research potential. Clin Epidemiol 2015;7:449-90.

22 Schmidt M, Pedersen L, Sørensen HT. The Danish civil registration system as a tool in epidemiology. Eur J Epidemiol 2014;29:541-9.

23 Statistik banken. Available: www.statistikbanken.dk

24 Vincent JL, de Mendonça A, Cantraine F, et al. Use of the SOFA score to assess the incidence of organ dysfunction/failure in intensive care units: results of a multicenter, prospective study. Working group on "sepsis-related problems" of the European Society of Intensive Care Medicine. Crit Care Med 1998;26:1793-800.

25 Vincent JL, Moreno R, Takala J, et al. The SOFA (sepsis-related organ failure assessment) score to describe organ dysfunction/ failure. on behalf of the Working group on sepsis-related problems of the European Society of intensive care medicine. Intensive Care Med 1996;22:707-10.

26 Singer M, Deutschman CS, Seymour CW, et al. The third International consensus definitions for sepsis and septic shock (Sepsis-3). JAMA 2016;315:801-10.

27 Guirgis FW, Puskarich MA, Smotherman C, et al. Development of a simple sequential organ failure assessment score for risk assessment of emergency department patients with sepsis. J Intensive Care Med 2017;45:088506661774128.

28 Seymour CW, Liu VX, Iwashyna TJ, et al. Assessment of clinical criteria for sepsis: for the third International consensus definitions for sepsis and septic shock (Sepsis-3). JAMA 2016;315:762-74.

29 Freund Y, Lemachatti N, Krastinova E, et al. Prognostic accuracy of Sepsis-3 criteria for in-hospital mortality among patients with suspected infection presenting to the emergency department. JAMA 2017;317:301-8.

30 Holm L, Brabrand M. The prognostic implication of the SOFA score on acutely admitted medical patients. Acute Medicine 2018;17:182-7.

31 Charlson ME, Pompei P, Ales KL, et al. A new method of classifying prognostic comorbidity in longitudinal studies: development and validation. Journal of chronic diseases 1987;40:373-83.

32 von Elm E, Altman DG, Egger M, et al. The strengthening the reporting of observational studies in epidemiology (STROBE) statement: guidelines for reporting observational studies. Int J Surg 2014;12:1495-9.

33 Vandenbroucke JP, von Elm E, Altman DG, et al. Strengthening the reporting of observational studies in epidemiology (STROBE): explanation and elaboration. Int J Surg 2014;12:1500-24.

$34 \mathrm{Kim}$ J, Kim K, Lee H, et al. Epidemiology of sepsis in Korea: a population-based study of incidence, mortality, cost and risk factors for death in sepsis. Clinical and Experimental Emergency Medicine 2019;6:49-63.

35 Mellhammar L, Wullt S, Lindberg Åsa, et al. Sepsis incidence: a population-based study. OFIDS 2016;3.

36 Yébenes JC, Ruiz-Rodriguez JC, Ferrer R, et al. Epidemiology of sepsis in Catalonia: analysis of incidence and outcomes in a European setting. Ann Intensive Care 2017;7:19.

37 Henriksen DP, Laursen CB, Jensen TG, et al. Incidence rate of community-acquired sepsis among hospitalized acute medical Patients-A population-based Survey*. Crit Care Med 2015;43:13-21.

38 Feigin VL, Lawes CMM, Bennett DA, et al. Worldwide stroke incidence and early case fatality reported in 56 population-based studies: a systematic review. Lancet Neurol 2009;8:355-69.

39 Kamalakannan S, Gudlavalleti ASV, Gudlavalleti VSM, et al. Incidence \& prevalence of stroke in India: A systematic review. Indian J Med Res 2017;146:175-85.

40 Yeh RW, Sidney S, Chandra M, et al. Population trends in the incidence and outcomes of acute myocardial infarction. $N$ Engl J Med 2010;362:2155-65.

41 Alzuhairi KS, Søgaard P, Ravkilde J, et al. Incidence and outcome of first myocardial infarction according to gender and age in Denmark over a 35-year period (1978-2012). Eur Heart J Qual Care Clin Outcomes 2015;1:72-8.

42 Raymond NJ, Nguyen M, Allmark S, et al. Modified sequential organ failure assessment sepsis score in an emergency department setting: retrospective assessment of prognostic value. Emerg Med Australas 2019;31.

43 Shapiro N, Howell MD, Bates DW, et al. The association of sepsis syndrome and organ dysfunction with mortality in emergency department patients with suspected infection. Ann Emerg Med 2006;48:583-90. 
44 Pedersen PB, Hrobjartsson A, Nielsen DL, et al. Prevalence and prognosis of acutely ill patients with organ failure at arrival to hospital: a systematic review. PLoS One 2018;13:e0206610.

45 Capan M, Hoover S, Ivy JS, et al. Not all organ dysfunctions are created equal - Prevalence and mortality in sepsis. J Crit Care 2018;48:257-62.

46 Linder A, Arnold R, Boyd JH, et al. Heparin-Binding protein measurement improves the prediction of severe infection with organ dysfunction in the emergency department. Crit Care Med 2015;43:2378-86.

47 Gaini S, Relster MM, Pedersen C, et al. Prediction of 28-days mortality with sequential organ failure assessment (SOFA), quick SOFA (qSOFA) and systemic inflammatory response syndrome (SIRS) - A retrospective study of medical patients with acute infectious disease. Int J Infect Dis 2019;78:1-7.

48 Quinten VM, van Meurs M, Wolffensperger AE, et al. Sepsis patients in the emergency department: stratification using the clinical impression score, predisposition, infection, response and organ dysfunction score or quick sequential organ failure assessment score? Eur J Emerg Med 2018;25:328-34.
49 Macdonald SPJ, Arendts G, Fatovich DM, et al. Comparison of PIRO, SOFA, and MEDS scores for predicting mortality in emergency department patients with severe sepsis and septic shock. Acad Emerg Med 2014;21:1257-63.

50 Levy MM, Artigas A, Phillips GS, et al. Outcomes of the surviving sepsis campaign in intensive care units in the USA and Europe: a prospective cohort study. Lancet Infect Dis 2012;12:919-24.

51 Levy MM, Fink MP, Marshall JC, et al. 2001 SCCM/ESICM/ACCP/ ATS/SIS international sepsis definitions conference. Intensive Care Med 2003;29:530-8.

52 Rahmatinejad Z, Reihani H, Tohidinezhad F, et al. Predictive performance of the SOFA and mSOFA scoring systems for predicting in-hospital mortality in the emergency department. Am J Emerg Med 2019;37:1237-1241

53 Grissom CK, Brown SM, Kuttler KG, et al. A modified sequential organ failure assessment score for critical care triage. Disaster Med Public Health Prep 2010;4:277-84.

54 Kakebeeke D, Vis A, de Deckere ER, et al. Lack of clinically evident signs of organ failure affects ED treatment of patients with severe sepsis. Int J Emerg Med 2013;6:4. 